\title{
Design of Multifunctional Bicycle Speedmeter Based on MCU
} XIN Haiyan $^{1}$, LI Chengxiang ${ }^{2}$, LU Yizhe $^{1}$, LI Yongmei $^{1}$, CHEN Yulin $^{1}$

1. Department of Electrical Engineering,Southeast University Chengxian College,Nanjing,Jiangsu 210088, China,

2. North information control group Co, Ltd. Nanjing,Jiangsu 211153,China

Keywords: Multifunctional; Bicycle Speedmeter; MCU

\begin{abstract}
Design a speed bicycle mileage meter, the system with MCU as the core processor, through the hall sensor acquisition bicycle riding speed, and with the GPS positioning module, through the MCU signal processing, speed, latitude and longitude of the display on the LCD. The experimental results show that the system is accurate, stable and reliable, and has a certain value of use.
\end{abstract}

\section{Introduction}

With the improvement of people's living standard, more and more people pay more attention to the quality of life, more and more contact with the world of his country, cultural blend, policy changes resulting in something had not paid attention to by people is more and more valued by people. Under such a background, the bicycle industry also revitalized, choice of more and more low carbon environmental protection of bicycle travel and exercise. At the same time, people also can by bike to challenge myself, and the corresponding, bicycle of a series of related derivatives will have a market value more and more. In this paper, we design a multifunctional bicycle mileage meter, which has the characteristics of small size, simple structure, light weight, stable and reliable operation and so on.

\section{Design of the Overall scheme of Bicycle Speedmeter}

\section{System design principles}

\section{(1) high reliability}

Reliability is a single-chip microcomputer system to using stable premise, on the bike in different environment can work steadily, bicycle used as different purposes may meet the rain, sediment and even some collision, when these conditions are met to work steadily. In order to improve the reliability, the high reliability of the components, the connection between the components should also be as stable and reliable.

(2) high cost performance

Microcontroller apart from a small volume, low power consumption and a obvious characteristic is the high cost, as the expected score of China's high-end products, work quality in a short time is hard to beat many foreign manufacturers, only the high price to broaden the market. The way to increase the cost of the price is to reduce the cost of hardware, such as simplifying the hardware circuit, using the software to replace the hardware.

(3) small size

As the table bicycle, in view of the limited space, and some of the need to speed the volume should be as small as possible. Now mainstream table volume are relatively small, easy to use, in order to achieve the small size effect, it is necessary to use more highly integrated components. At the same time, we walk the line, the hardware circuit should be as concise as possible.

\section{system overall plan}

The system needs to be completed as follows: 
(1) the data collected by GPS UBLOX- and the real-time pulse acquisition by the Holzer device, which is processed by the single chip microcomputer to realize the continuous measurement of the speed and the real-time update of the location information;

(2) the equipment should react in a timely manner, the accuracy is relatively high;

(3) software design should be streamlined, the implementation of the speed to be fast;

The system block diagram is shown in Figure 1.

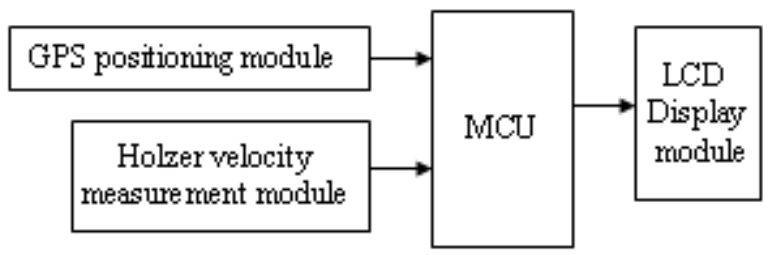

Figure 1. The system block diagram

\section{Design of the hardware system of Bicycle Speedmeter}

\section{Master control module STC89C52}

STC89C52 is a kind of low power, high performance CMOS8 bit microcontroller produced by STC company. It has Flash memory which can be programmed in the system. STC89C52 uses the classic MCS-51 kernel, but made a lot of improvements that make the chip has a traditional 51 do not have the function. On a single chip, with a smart 8 bit CPU and the system can be programmed Flash, making STC89C52 for many embedded control applications to provide a highly flexible, ultra efficient solution.

\section{Clock circuit}

The design uses the external clock, the clock frequency is $11.059 \mathrm{kHz}$. By reason of $11.0592 \mathrm{k}$ crystal is 52 single-chip timer to. Baud rate generator with 52 single-chip timer to do, if $11.059 \mathrm{khz}$ crystal. According to the formula count down timer needs to set the values are integers; if $12 \mathrm{khz}$ crystal, the baud rate is biased, for example, 9600, use the timer to take the FD, actual baud rate to 10000. Schematic diagram shown in figure 2.

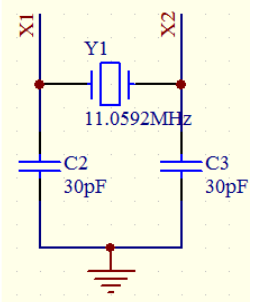

Reset Circuit

Fig. 2 Schematic diagram of clock circuit

When there is an error in the table do not work or the work can be through the reset circuit one-time correction, which can be normally used, hardware hesitate instability and software vulnerabilities, although the development will try to avoid such things happen, but still there will be inevitable, that table is to be sustained, a reset button is very important. Schematic diagram shown in figure 3.

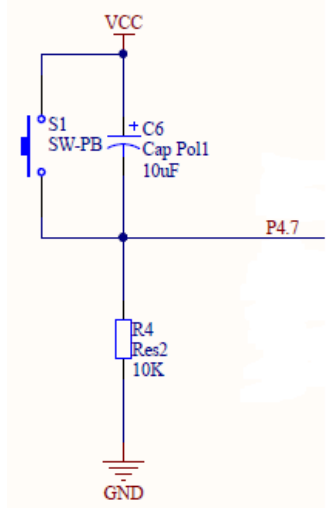

Fig.3 schematic diagram of reset circuit 


\section{Holzer velocity measurement module}

If the Holzer element integrated switch is arranged regularly on the object according to the predetermined position, the permanent magnet which is mounted on the moving object can be measured from the measuring circuit to get the pulse signal when the permanent magnet is arranged on the moving object. The displacement of the moving object can be sensed according to the pulse signal column. If the number of pulses emitted per unit time is measured, the movement speed of the pulse can be determined [4]. Holzer velocity module schematic diagram shown in figure 4.

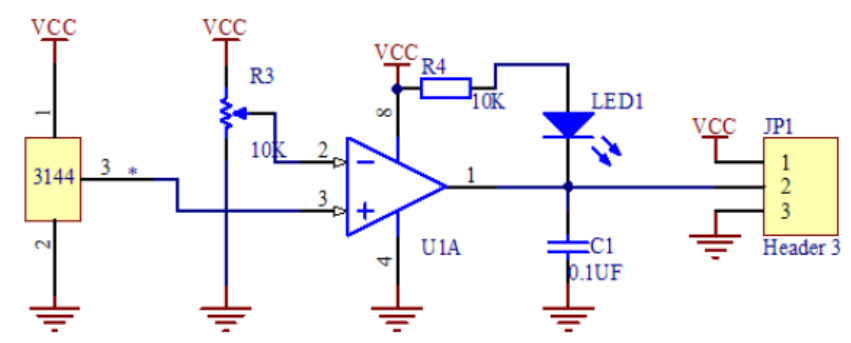

\section{GPS module}

Fig. 4 Schematic diagram of Holzer velocity measurement module

The GPS module is actually a receiver chip, through constant accept different satellites to send over the time and location information, collected and sent to the CPU core, when the mobile GPS module, the received data also produce change subsequently, CPU through production when copied into the algorithm will accept the position in time is calculated, their position and velocity information. GPS module schematic as shown in figure 5.

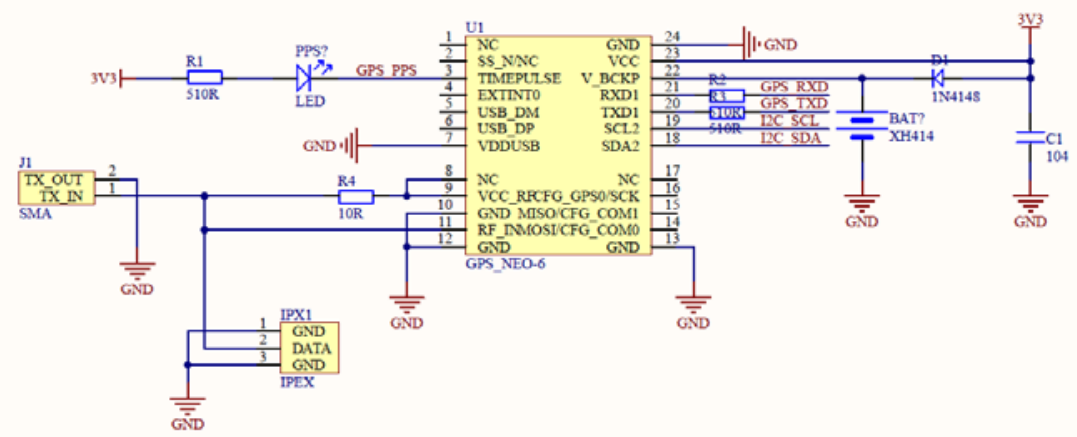

\section{LCD 12864 display module}

Fig. 5 schematic diagram of GPS

12864 is a general term that is composed of $128 * 64$ points, this time with LCD 12864 parallel port communication, communication programming more convenient and LCD 12864 compared to LCD 1602, in addition to display content more, also comes with a Chinese font, use rise very convenient. Contrast to the TFT LCD, less power consumption, although cannot display color and more information, but as a positioning end tables and display, which can display the information has enough design. Schematic diagram shown in figure 6. 


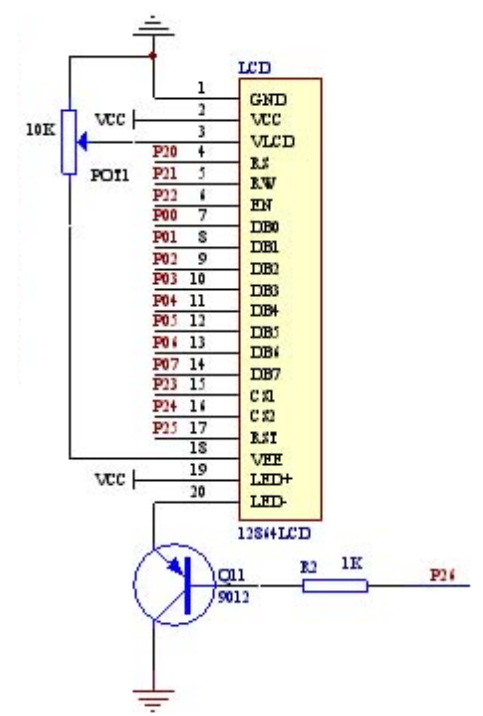

Fig. 6 schematic diagram of LCD 12864

\section{Design of the software system of Bicycle Speedmeter}

\section{Main Program}

First initialize the display module, then set display module to the ordinary mode and when to collect "GPRMC" the frame data enable interrupt, processing of GPS signal, converts UTC date for the Beijing time, the date of the conversion to the corresponding ASCII code, latitude and longitude also goes through a similar process, and then are sent to the 12864 display. The data received by the Holzer component is also shown through LCD 12864 after the data processed by function. Design ideas as shown in Figure7 .

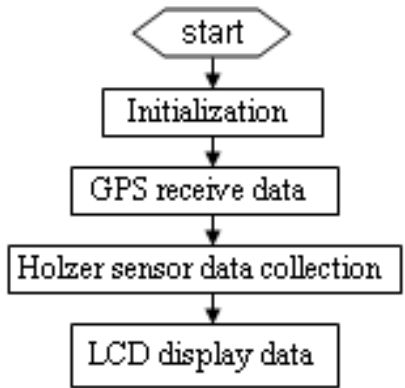

Fig. 7 flow chart of the main program

\section{Holzer components processing program}

Regulation of the microcontroller control word, the total open interrupt, serial port interrupt enable, allow timer T1 interrupt, set timer T1 in mode 2 interrupts the baud rate, set SMOD $=0$, set the baud rate is 9600, T1 timer operation control bits. Due to the magnet swept across the hall element for a period of time, so the need for a similar to elimination Buffeting delay procedures, when the change in level of the hall element, to generate an interrupt, the program from the main program jump to the hall element program, by a variable by counting and timing, so as to remember the time interval between two high level, and then take the wheel diameter can be obtained by the time distance, a unit conversion can be obtained per hour. Then the data is processed to get its corresponding ASCII code, which is displayed on the LCD 12864. The flow chart is shown in figure8. 


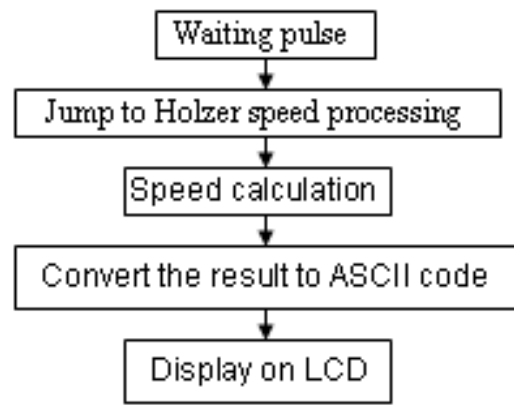

\section{GPS data analysis program}

Fig. 8 flow chart of Holzer component processing program

Whenever the receiving data is from the main program into the GPS data receiving program, data receiving end after the switch to the main program and ES is set to 1, then transferred to the main program, through the fixed algorithm for GPS acquisition to the information to calculate the rider usually can easily read the information. Because of the need for positioning information through 12864 LCD display, and therefore have income data conversion the according to the character of LCD 12864 through 12864 LCD display. Process as shown in Figure 9.

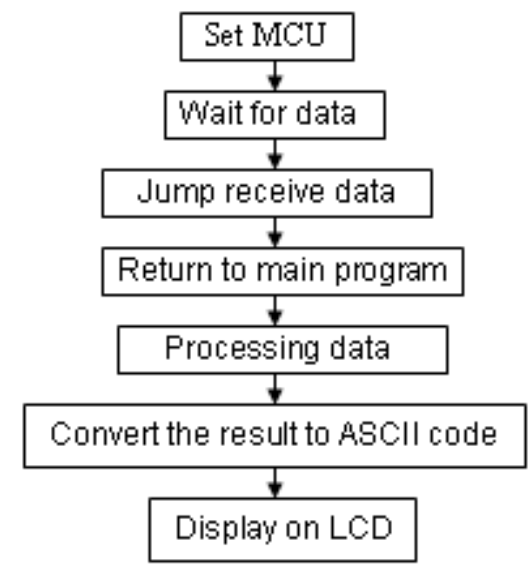

Fig. 9 flow chart of GPS data analysis

\section{Conclusion}

In today's society, energy resources are more scarce, all over the world, including China to advocate green travel, travel by bike more and become a kind of fashion, style, and even a kind of attitude to life, with the increasing popularity and development of the bicycle, the associated with a number of supporting equipment will be booming, stopwatch is one of them. This design takes the single chip microcomputer as the core processor, designs a kind of multi function bicycle mileage meter, and carries the GPS positioning module, realizes the localization function. The experimental results show that the system has the characteristics of small size, light weight, low power consumption, reliable operation and so on, which has a certain value of application.

\section{References}

[1] Zhao Yan, Li Wei. Design of GPS positioning display system based on STC MCU [J]. Journal of Nanjing Institute of Industry Technology, 2014, 4 (4): 1671-4644.

[2] Tian Wei, Zhang Zunyang. Wang Fuping et al. Multifunction bicycle speed measurement system [J]. electronic world, 2014 (24).

[3] Zhao Xiaolan Hu syndrome. Wang Peikun et al. Multifunctional bike design [J]. electronic test, $2013(20)$. 